\title{
Modelagem empírica de funções multivariáveis por redes neurais artificiais
}

\author{
Cansian, A. B. M. ${ }^{1 *}$; Costa, A. O. S. ${ }^{2}$; Costa Jr, E. F. C. ${ }^{2}$ \\ 1 Curso de Graduação em Engenharia Química, Universidade Federal do Espírito Santo, Alegre, ES, Brasil \\ 2 Programa de Pós-Graduação em Engenharia Química e Graduação em Engenharia Química \\ Departamento de Engenharia Rural, Universidade Federal do Espírito Santo, Alegre, ES, Brasil \\ *e-mail: ana_barbara_moulin@hotmail.com
}

\section{Resumo}

A modelagem é uma importante ferramenta matemática utilizada na previsão de resultados em diferentes problemas na engenharia, na física, química e em outras áreas que utilizam desse artifício para prever o comportamento de determinadas variáveis, gerando um modelo empírico ou não. Há diversas formas de se obter um modelo empírico, entre elas destacam-se para o trabalho em questão as redes neurais artificiais. Essas redes seguem a mesma lógica de aprendizagem de um cérebro humano, tirando conclusões através da experiência obtida em observações. Com isso, o presente trabalho teve por objetivo analisar aproximações de funções de duas variáveis independentes utilizando de um número de dados fixo em um intervalo definido, de forma a obter modelos totalmente empíricos. O parâmetro variado no trabalho foi o número de neurônios disponibilizados às redes para cada aproximação (1 a 5 neurônios). Foram comparados os módulos dos erros de treinamento e validação de cada modelo obtido. Além da análise numérica dos erros, foram realizadas análises gráficas comparando o comportamento dos modelos gerados com o das funções a serem aproximadas. A partir dos testes realizados e das análises gráficas e numéricas, pretendeu-se concluir em quais situações as redes obtiveram maior eficiência nas aproximações das diferentes funções.

Palavras chaves: Modelagem Empírica, Redes Neurais Artificiais, Funções Matemáticas.

\begin{abstract}
Modeling is an important mathematical tool used in forecasting results in different problems in engineering, physics, chemistry and other areas that use this device to predict the behavior of certain variables, generating an empirical model or not. There are several ways to obtain an empirical model, among these ways stand out the artificial neural networks for this job. These networks follow the same logic learning of a human brain, drawing conclusions from the experience gained from observations. Thus, this study aimed to analyze approximations of functions with two independent variables using a fixed number of data at a set interval, to obtain fully empirical models. The varied parameter in the study was the number of neurons available to the networks for each approximation (1-5 neurons). The modules of the errors of training and validation of each model obtained were compared. In addition to the numerical error analysis, graphical analyzes comparing the behavior of the models generated with the functions to be approximated were held. From the tests, graphic analysis and numerical analysis, the study intended to conclude in which situations the networks had higher efficiency in the approximations of the different functions.
\end{abstract}




\section{Introdução}

A modelagem é uma ferramenta amplamente utilizada na matemática, física, engenharia, entre outras áreas para predizer o comportamento de uma ou mais variáveis em estudo. Modelo é uma descrição de um processo (comportamento) real ou não que se pretende trabalhar por meio de relações matemáticas ou lógicas [1]. Um modelo em cujo desenvolvimento somente foram empregadas informações experimentais, sem informações teóricas sobre o fenômeno estudado, é denominado modelo empírico. Há diversas formas de se obter modelos empíricos sendo a modelagem por redes neurais artificiais adotada neste trabalho.

As redes neurais artificiais têm seu funcionamento baseado na habilidade de processamento e aprendizagem do cérebro humano, ou seja, a absorção de informações se dá com base na observação comportamental e na experiência obtida por meio dessa. Sendo assim, elas têm capacidade de resolver problemas com diferentes graus de complexidade, visto que as previsões se dão através das observações de comportamento. A aprendizagem das redes ocorre de forma a alterar, por meio de um algoritmo, seus pesos sinápticos, reduzindo os erros do modelo. O neurônio é a unidade de processamento básica das redes [2]; [3].

O conjunto de dados em análise é divido, de forma aleatória, em três outros: treinamento, validação e teste. Os dados de treinamentos transmitem às redes o comportamento geral do modelo. Já o conjunto de validação faz com que o modelo generalize e não apenas tenha enfoque nos dados de treinamento. Por fim, os testes avaliam os modelos e determinam quando os processamentos terminam, ou seja, quando a média dos erros chega a um limite e não se diminui mais, levando então à resposta final: o modelo [2].

As respostas e análises apresentadas neste trabalho são parte dos resultados obtidos através do projeto de pesquisa intitulado "Princípios de Modelagem Matemática pela Aplicação de Redes Neurais Artificiais" aprovado na Chamada No 18/2013 MCTI/CNPq/SPM-PR/Petrobras - Meninas e Jovens Fazendo Ciências Exatas, Engenharias e Computação.

\section{Materiais e Métodos}

O trabalho teve por objetivo utilizar modelagem empírica por meio de redes neurais artificiais para predizer 0 comportamento de algumas funções matemáticas de duas variáveis independentes. Os treinamentos foram realizados por meio do módulo de redes neurais do software STATISTICA. No total, foram gerados 144 dados no intervalo de $-5 \leq x \leq 5$ e $-5 \leq y \leq$ 5 para serem aplicados em todas as aproximações realizadas.
Os neurônios são unidades importantes no processamento das redes. Neste trabalho objetivou-se variar o número de neurônios em cada aproximação e analisar o comportamento dos módulos dos erros de treinamento e validação, frente às complexidades de cada função. As análises também acontecem por meio de gráficos, de forma a visualizar as discrepâncias dos modelos em relação às funções originais.

\section{Resultados e Discussão}

Ao decorrer do trabalho, os modelos foram gerados a partir de aproximações de funções de várias variáveis, mais especificamente, funções com duas variáveis independentes. Neste caso, é possível a visualização e comparação dos resultados por meio das representações gráficas.

O software de trabalho possui diferentes parâmetros que podem ser variados a fim de se obter melhores modelos como resposta. Assim, nas observações realizadas variou-se o número de neurônios nas redes neurais (1 a 5 neurônios) a fim de observar em quais casos obteve-se menores erros nas aproximações. Em alguns casos, poucos neurônios foram necessários para encontrar bons modelos, em outros foi preciso um número maior. Nenhum erro foi adicionado aos dados para aproximação.

A primeira função utilizada nos treinamentos foi $f(x, y)=$ $3 x-2 y$. A representação gráfica dessa função é observada na figura 1.

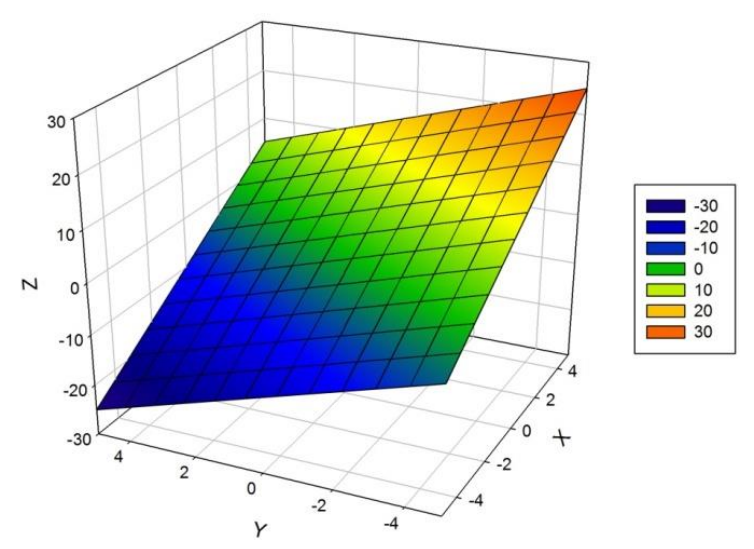

FIGURA 1 - Representação gráfica da função $f(x, y)=3 x-2 y ;(-5 \leq x \leq$ 5) ; $(-5 \leq y \leq 5)$. Fonte: Autores.

Para cada valor do número de neurônios, foram geradas dezenas ou centenas de redes, sendo selecionada a com melhor desempenho. Avaliação de desempenho foi baseada na média dos módulos dos erros de validação e treinamento. Para a primeira função trabalhada os erros encontram-se dispostos na tabela 1. 
TABELA 1 - Média dos Módulos dos Erros de Treinamento e de Validação para função $(x, y)=3 x-2 y$, para dados gerados sem erro, ($5 \leq x \leq 5) ;(-5 \leq y \leq 5)$

\begin{tabular}{ccc}
\hline $\begin{array}{c}\text { Erro de } \\
\text { Treinamento }\end{array}$ & $\begin{array}{c}\text { Erro de } \\
\text { Validação }\end{array}$ & $\begin{array}{c}\text { Número de } \\
\text { Neurônios }\end{array}$ \\
\hline 0,003166 & 0,003635 & 1 \\
0,001821 & 0,002123 & 2 \\
0,001451 & 0,002117 & 3 \\
0,001299 & 0,001534 & 4 \\
0,001471 & 0,001466 & 5 \\
\hline
\end{tabular}

Fonte: Autores

Em teoria, sabe-se que o aumento no número de neurônios para a resolução de um problema aumenta o nível de "facilidade" com que as redes obtêm os resultados. Aproximações realizadas com funções de uma única variável independente concluíram que o número de neurônios interfere nos resultados obtidos [4]. Assim, o erro de validação apresentou um comportamento já esperado, uma vez que seu valor diminui à medida que o número de neurônios aumentou. $\mathrm{O}$ erro de treinamento dos modelos seguiu o mesmo comportamento, à exceção do resultado obtido ao se passar de 4 para 5 neurônios. Ainda assim, percebe-se que todos os erros são pequenos comparados entre si, visto que a função pode ser classificada como simples e de fácil aproximação, uma vez que seu comportamento é linear. Com os resultados do modelo gerado com apenas um neurônio, nesse caso, é possível representar a função com precisão. $\mathrm{Na}$ figura 2 está representado o modelo obtido.

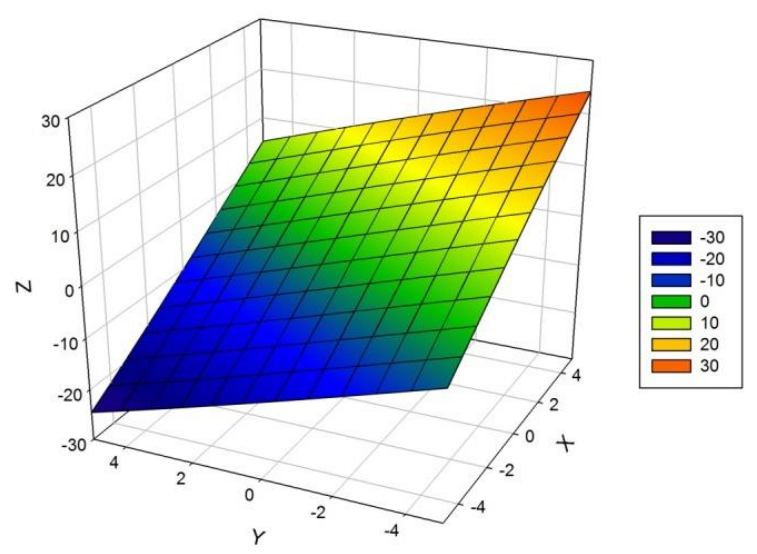

FIGURA 2 - Representação gráfica do modelo aproximado com 1 neurônio para função $f(x, y)=3 x-2 y ;(-5 \leq x \leq 5) ;(-5 \leq y \leq 5)$ (dados gerados sem erros). Fonte: Autores.
Outra função aproximada foi $f(x, y)=3 x^{2}+2 y^{2}$. Esta função está graficamente representada na figura 3 , dentro do intervalo considero e sem erros adicionados.

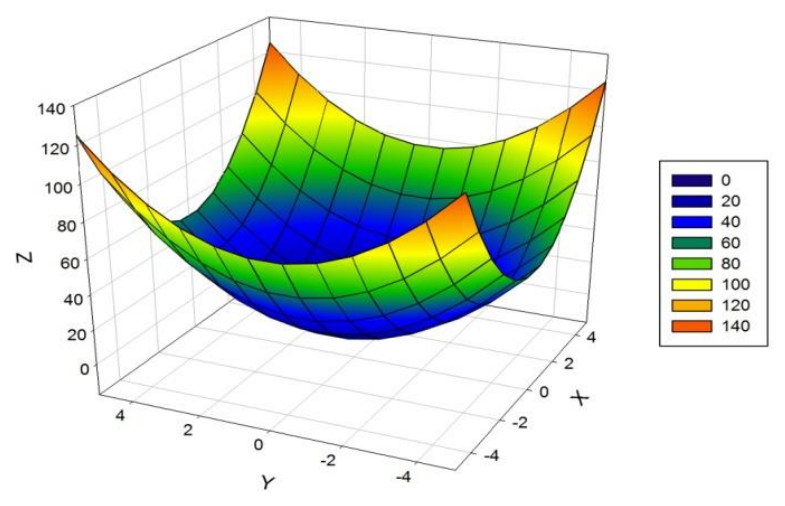

FIGURA 3 - Representação gráfica da função $f(x, y)=3 x^{2}+2 y^{2},(-5 \leq x$ $\leq 5) ;(-5 \leq y \leq 5)$. Fonte: Autores.

Foram adotados os procedimentos anteriores para encontrar os modelos variando o número de neurônios. Os erros de treinamento e validação dos diferentes modelos encontrados estão na tabela 2.

TABELA 2 - Média dos Módulos dos Erros de Treinamento e de Validação para função $(x, y)=3 x^{2}+2 y^{2}$, para dados gerados sem erro, $(-5 \leq x \leq 5) ;(-5 \leq y \leq 5)$

\begin{tabular}{ccc}
\hline $\begin{array}{c}\text { Erro de } \\
\text { Treinamento }\end{array}$ & $\begin{array}{c}\text { Erro de } \\
\text { Validação }\end{array}$ & $\begin{array}{c}\text { Número de } \\
\text { Neurônios }\end{array}$ \\
\hline 0,226092 & 0,236125 & 1 \\
0,136521 & 0,147730 & 2 \\
0,014052 & 0,018087 & 3 \\
0,011849 & 0,014143 & 4 \\
0,009801 & 0,012262 & 5 \\
\hline Fonte: Autores. & & \\
\hline
\end{tabular}

O comportamento dos modelos aproximados representados na tabela 2 exprime a mesma sequência da aproximação anterior, onde há decaimento da média dos módulos à medida que o número de neurônios aumenta. A figura 4 representa graficamente os modelos aproximados com 1, 2, 3 e 4 neurônios. 
(a)

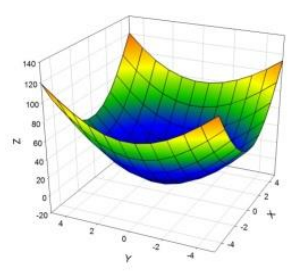

(c)

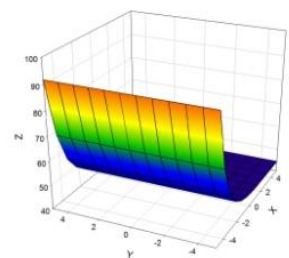

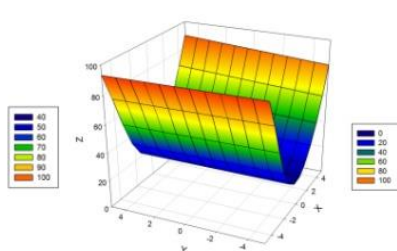

(b)

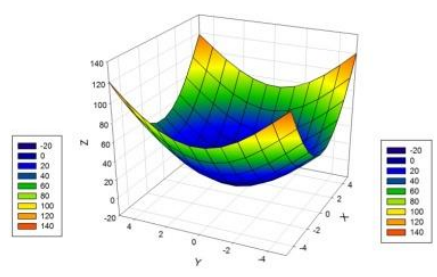

(d)
FIGURA 4 - Representação gráfica dos modelos aproximados para função $f(x, y)=3 x^{2}+2 y^{2} ;(-5 \leq x \leq 5) ;(-5 \leq y \leq 5)$ (dados gerados sem erros). (a) - Modelo com 1 neurônio. (b) - Modelo com 2 neurônios. (c) - Modelo com 3 neurônios. (d) - Modelo com 4 neurônios. Fonte: Autores.

Pela figura 4 é nítida a percepção da importância do número de neurônios nas aproximações. No modelo aproximado com 1 neurônio na figura 4.(a) percebe-se grande diferença quando comparado ao gráfico da função representado na figura 3. Assim, também, comparam-se os gráficos seguintes acompanhando a evolução nos modelos obtidos em função do número de neurônios. Por fim, a figura 5 representa o melhor modelo encontrado, com 5 neurônios.

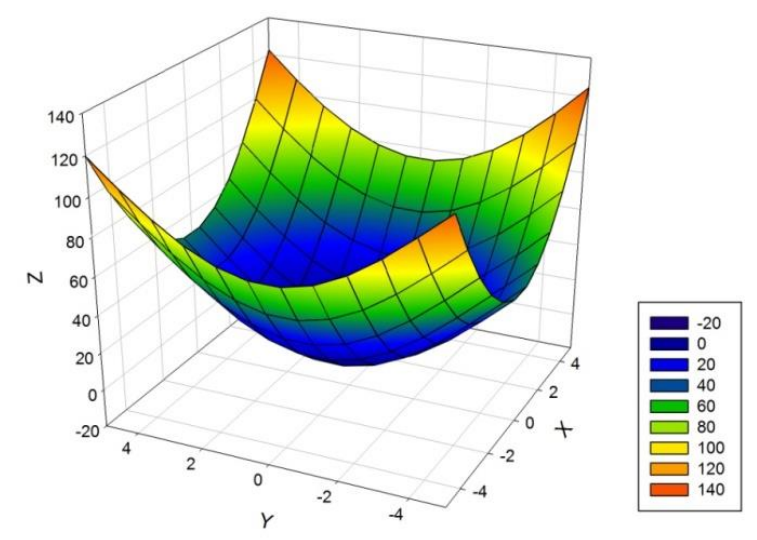

FIGURA 5 - Representação gráfica do modelo aproximado com 5 neurônios para função $f(x, y)=3 x^{2}+2 y^{2} ;(-5 \leq x \leq 5) ;(-5 \leq y \leq 5)$ (dados gerados sem erros). Fonte: Autores.

A função $f(x, y)=[(\operatorname{sen}(x) \operatorname{sen}(y)] /(x y)$ também foi observada nas aproximações. Apenas olhando para a representação algébrica da função nota-se sua complexidade. Graficamente é possível perceber de forma mais convincente as discrepâncias da função (analise a figura 6). É importante ressaltar que a essa função não está definida nos pontos em que $x$ ou $y$ ou $x$ e y são iguais a zero (muito embora o limite desta função, para x e/ou y tendendo a zero, possa ser calculado). Este problema foi contornado pois na geração dos dados de treinamento e validação, não foram empregados valores nulos para $\mathrm{x}$ ou $\mathrm{y}$.

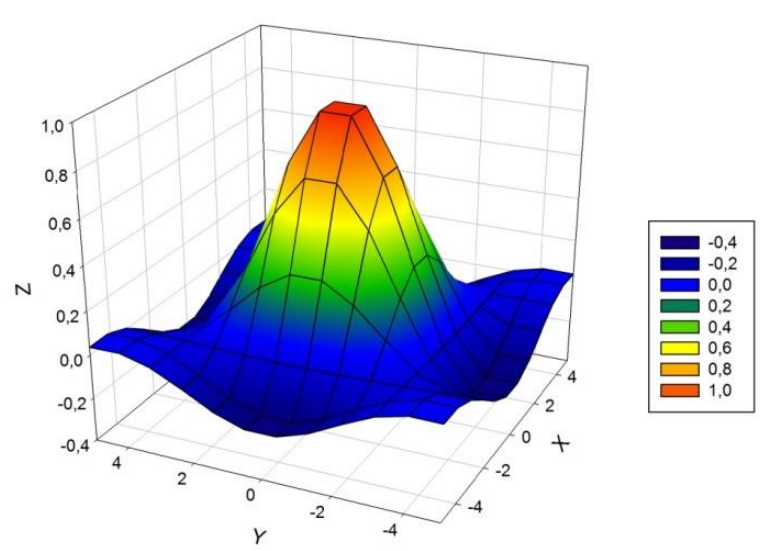

FIGURA 6 - Representação gráfica para função $f(x, y)=$ $[(\operatorname{sen}(x) \operatorname{sen}(y)] /(x y) ;(-5 \leq x \leq 5) ;(-5 \leq y \leq 5)$. Fonte: Autores.

As aproximações realizadas para a função representada na figura 6 também foram analisadas variando o número de neurônios. A média dos módulos dos erros de treinamento e validação encontra-se na tabela 3 .

TABELA 3 - Média dos Módulos dos Erros de Treinamento e de Validação para função $f(x, y)=[(\operatorname{sen}(x) \operatorname{sen}(y)] /(x y)$, para dados gerados sem erro, $(-5 \leq x \leq 5)$; $(-5 \leq y \leq 5)$.

\begin{tabular}{ccc}
\hline $\begin{array}{c}\text { Erro de } \\
\text { Treinamento }\end{array}$ & $\begin{array}{c}\text { Erro de } \\
\text { Validação }\end{array}$ & $\begin{array}{c}\text { Número de } \\
\text { Neurônios }\end{array}$ \\
\hline 0,194170 & 0,200821 & 1 \\
0,190141 & 0,195278 & 2 \\
0,113918 & 0,134376 & 3 \\
0,110200 & 0,126034 & 4 \\
0,104044 & 0,123872 & 5 \\
\hline Fonte: Autores. & &
\end{tabular}

Com base na tabela 3 , conclui-se mais uma vez o comportamento geral dos modelos. À medida que o número de neurônios aumenta, os módulos dos erros de treinamento e validação diminuem, visto como a complexidade para resolução do problema também aumenta. Porém, nem mesmo aumentando o número de neurônios até 5 foi suficiente para resolver 0 problema com eficiência, isto pode ser percebido através da figura 7, onde encontram-se os gráficos dos modelos aproximados com 2, 3, 4 e 5 neurônios. 


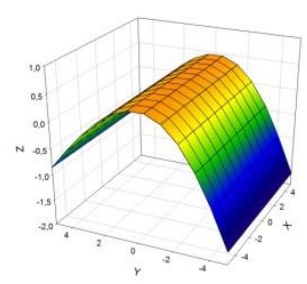

(a)

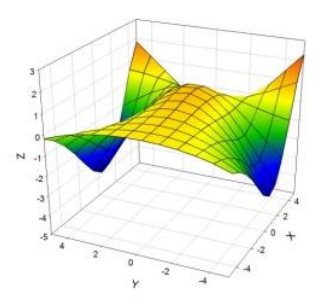

(c)

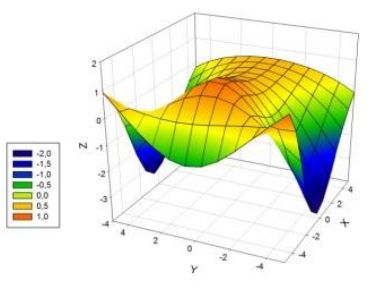

(b)

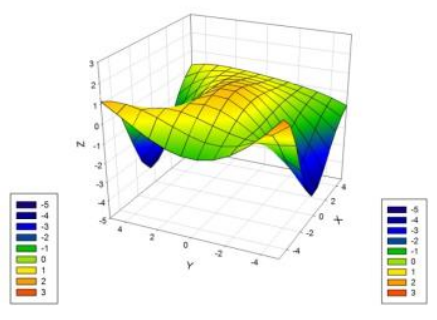

(d)
FIGURA 7 - Representação gráfica dos modelos aproximados para função $f(x, y)=[(\operatorname{sen}(x) \operatorname{sen}(y)] /(x y) ;(-5 \leq x \leq 5) ;(-5 \leq y \leq 5)$ (dados gerados sem erros). (a) - Modelo com 2 neurônios. (b) - Modelo com 3 neurônios. (c) - Modelo com 4 neurônios. (d) - Modelo com 5 neurônios. Fonte: Autores.

A complexidade da função $f(x, y)=[(\operatorname{sen}(x) \operatorname{sen}(y)] /(x y)$ é reinterada analisando-se a figura 7 . Nenhum dos 4 modelos encontrados predizem a resposta aproximada da função real. O comportamento encontrado é um caminho, mas está longe do valor da função.

As tentativas de encontrar uma boa resposta prosseguiram, aumentando-se o número de neurônios até que um resultado encontrado fosse razoável. Entre muitos testes e com 0 aumento gradativo da complexidade nas aproximações chegou-se em um número de 10 neurônios, e o resultado encontrado pode ser analisado graficamente pela figura 8 .

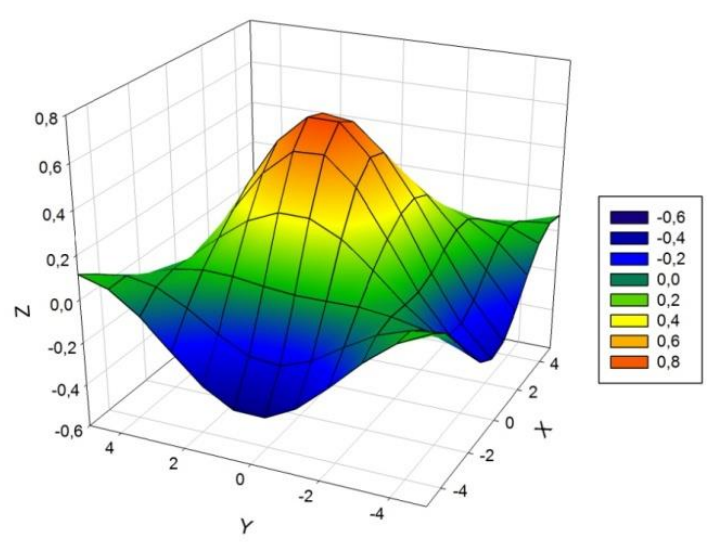

FIGURA 8 - Representação gráfica do modelo aproximado com 10 neurônios para função $f(x, y)=[(\operatorname{sen}(x) \operatorname{sen}(y)] /(x y) ;(-5 \leq x \leq 5) ;(-5 \leq y \leq$ 5) (dados gerados sem erros). Fonte: Autores.
Apesar de o resultado encontrado ser considerado muito bom para as condições de trabalho, ainda encontra-se distante dos valores reais da função. Porém para determinadas finalidades em problemas reais, o resultado poderia ser interessante, já que o comportamento qualitativo da função foi bem representado pelo modelo.

\section{Conclusão}

As funções trabalhadas ao longo das aproximações possuem comportamentos diferentes assim como graus de complexidade distintos. Com isso, foi possível observar como as redes neurais artificiais geram modelos que se adéquam facilmente ou não às funções dadas. Como visto, o número de neurônios usados em cada aproximação foi um fator determinante e de suma importância para obter bons resultados quando os problemas ficavam mais complexos. Em funções mais simples um número reduzido de neurônios é necessário para respostas eficientes, já em funções complexas aumenta-se o número de neurônios até se obter um resultado próximo ao desejado. Assim conclui-se que o aumento do número de neurônios contribui para geração de modelos empíricos satisfatórios.

\section{Agradecimentos}

Os autores agradecem ao CNPq pelo apoio financeiro e pelas bolsas para discentes, processo $n^{\circ}$ 420147/2013-6, e pela bolsa de produtividade DTI e à FAPES pela bolsa de pesquisador capixaba.

\section{Referências}

[1] FREITAS Filho, P. J., Introdução à Modelagem e Simulação de Sistemas com aplicações em Arena. $2^{\underline{a}}$ ed. Visual Books. Florianópolis, 2008. Pag. 44-45.

[2] HAYKIN, S., Redes Neurais: Princípios e Prática. 2ª ed. Porto Alegre, RS, 2008.

[3] BITTENCOURT, G., Inteligência Artificial. $3^{\underline{a}}$ ed. Revisada. Editora da UFSC. Florianópolis, 2006.

[4] CANSIAN, A. B. M.; COSTA JUNIOR, E. F.; COSTA, A. O. S., Utilização de redes neurais artificiais como estímulo ao aprendizado de matemática. Enciclopédia Biosfera, v. 10, p. 2567-2580, 2014. 